\title{
Global and local gravitational redshifts in cosmology and their consequences for theory and observations
}

\begin{abstract}
Zahid Zakir
Abstract

A consistent theory of gravitational redshift in cosmology (GRC) is formulated. The global GRC arises due to weakening of gravitational time dilation due to decreasing of matter density during the propagation time of photons. In the expanding world the local GRC arises due to the weakening of gravity of the sphere between observer and source, since photons emitted at a smaller radius arrive at a larger one. In static world there is no GRC at the exchange of photons at the periphery of this sphere. In any case photons from observer to source have the same GRC as photons from source to observer, which is in agreement with the cosmological principle. Consequences of the local and global GRC for cosmological models and their parameters, as well as corrections to data on distant objects and CMB, are considered. In Appendix the inconsistency of two former treatments of the gravitational frequency shift in cosmology is shown. They: a) did not take into account the global GRC; b) derived the local GRC not from the field of the sphere between the source and observer, but from the field of spheres around one of them; c) contradicted each other (the signs of shifts are opposite); d) violated cosmological principle (changing the propagation direction changes the sign of shift) and e) were based on the delusion that the Friedmann model supposedly contains the gravitational shift.
\end{abstract}

Keywords: cosmological models, cosmological redshift, Doppler effect, cosmological principle, CMB.

\section{Содержание}

Introduction

1. The theory of gravitational frequency shift in cosmology ............................................. 3

1.1. A new method for accounting for gravity in cosmology and two types of GRC.........3

1.2. The global GRC. ......................................................................................... 4

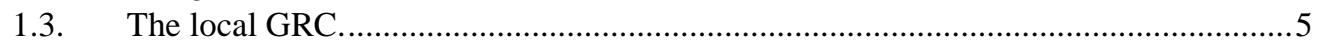

1.4. Total GRC and its combinations with other types of redshift. ...............................6

2. Consequences of two types of GRC in cosmology ........................................................... 7

2.1. Consequences of GRC for cosmological models and interpretation of data ...............7

2.2. Consequences of GRC for CMB and the early universe ................................... 7

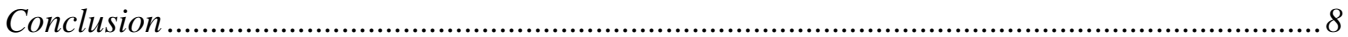

Appendix. Inconsistency of two former treatments of gravitational shift in cosmology............... 9

References.....

\section{Introduction}

Two effects were usually considered as the main mechanisms for the frequency shift of photon in cosmology - the Doppler effect and stretching of wavelengths during expansion of space, which have a linear part in the formula for the dependence of redshifts on distance. For more distant objects, one should also take into account the third mechanism - the gravitational

${ }^{1}$ Center for Theoretical Physics and Astrophysics, Tashkent Uzbekistan, zzakir@qgph.org, ORCID 
shift of frequencies, the contribution of which is very small for close objects, but it grows quadratically with distance and becomes significant for distant objects. However, two previous attempts to take it into account turned out to be erroneous, and the purpose of the present paper is to formulate a consistent theory of gravitational frequency shift in cosmology.

The gravitational frequency shift, which in the first approximation there is already in Newtonian gravity, was predicted by Einstein in the framework of general relativity [1] and was later confirmed in many experiments. It was also experimentally confirmed the physical interpretation of the gravitational shift in general relativity as a result of the difference in the rate of local proper times near the source and the observer [2].

Therefore, being one of the basic effects of general relativity, gravitational shift had to be taken into account in cosmology. However, in the initial period of the formation of relativistic cosmology, it was ignored due to the smallness of its contribution for close objects. When more accurate data on distant objects appeared, misconceptions in the treatment and errors in calculating the contribution of gravitational shift delayed its consideration in both theoretical and observational cosmology. As a result, predictions of models for distant objects were made without taking into account this effect, and observational data were used to test these models also without correcting for this effect. All this made both theory and interpretation of data concerning distant sources insufficiently adequate to reality.

Early treatments of gravitational shift in cosmology used two methods. In the first one, Bondi [3] considered the propagation of photons in a sphere around the source (sphere B in Fig. 1) and predicted gravitational redshift for photons arriving at the observer. In the second method, Zeldovich and Novikov [4] (see also [5,6]) considered the photons in a sphere around the observer (sphere A in Fig. 1), and predicted a gravitational violetshift for the same photons.

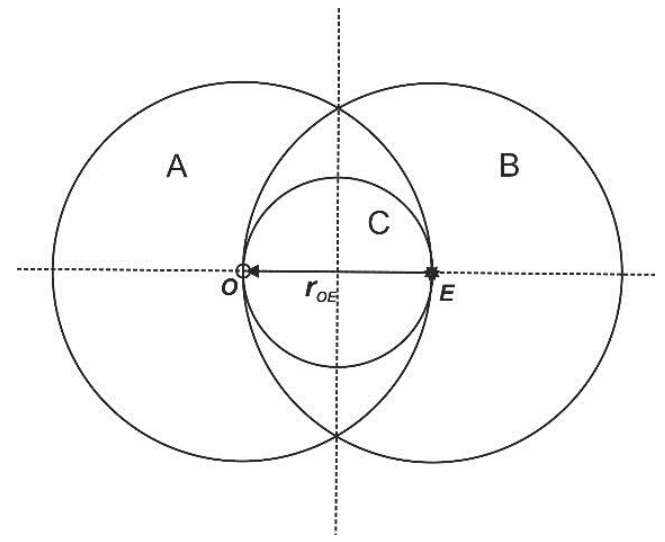

Fig. 1. Photons from the source E, coming to the observer $\mathrm{O}$, in the gravitational field of three spheres A, B, C of three methods.

a) Sphere $A$ around the observer with radius up to the source $r_{O E}$ (first method [3]).

b) Sphere B around the source with the same radius (second method [4]).

c) Sphere $\mathrm{C}$ between the observer and the source with radius $r_{O E} / 2$ (third method - the present paper).

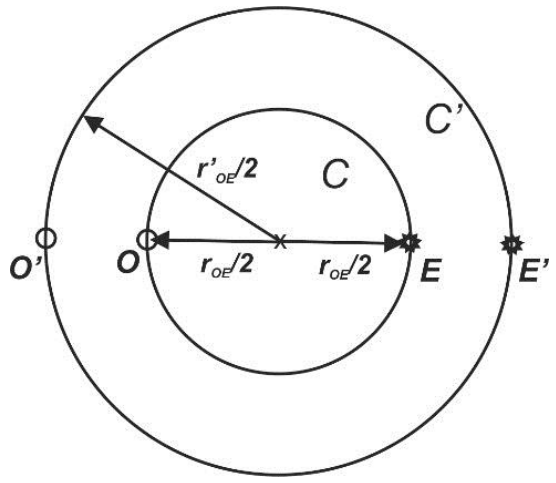

Fig. 2. Photons in the gravitational field of sphere $\mathrm{C}$ between the source and the observer in the expanding world. Photons are emitted by the source $\mathrm{E}$ at the radius $r_{O E} / 2$, and are received by the observer $\mathrm{O}^{\prime}$ at the larger radius $r_{O E}^{\prime} / 2$. The mass of the sphere $\mathrm{C}$ does not change during expansion, and therefore the gravitational field at its periphery was previously stronger, which leads to the gravitational redshift of the frequencies of photons emitted in early epochs with respect to photons emitted at the time of reception.

Moreover, each of these methods was internally contradictory, since they led to the inequality of two objects in the universe, violating the cosmological principle from which they proceed. If the first two effects, Doppler shift and stretching, are relative and the same for two objects exchanging photons, the gravitational displacement turned out to be asymmetric and 
changed sign at changing the propagation direction of photons. As it is well known, photons from a satellite to a ground observer come with violetshift, and from a ground source to the satellite - with a redshift. Therefore, in the first treatment, photons from the source to the observer come with a gravitational redshift, and photons from the observer to the source - with violetshift, which was noted by Bondi [3]. In the second treatment, everything is the same, but with the opposite sign.

Another internal contradiction was the fact that the gravitational shift was calculated in the Newtonian approximation, where it supplemented the Doppler shift, but it was considered as implicitly taken into account in the formulas of the Friedmann model. As a result, it was argued that in the relativistic theory this shift should not be considered additionally [4]. This contradicted the fact that all the basic relations of the Friedmann model exactly follow from the Newtonian approximation.

Thus, the two former treatments of gravitational shift in cosmology [5,6] were internally contradictory and contradicted each other, giving shifts of opposite sign. Details of these two treatments and their criticism are presented in the Appendix.

In the present paper, a third consistent method is formulated that makes it possible to take into account the gravitational shift in cosmology without the indicated contradictions and its consequences are studied. At the first stage, it is proved that the local gravitational redshift in cosmology (local GRC) is the result of the influence of gravity of matter in the sphere between the observer and the source, centered in the middle of the distance to the source (sphere $\mathrm{C}$ in Fig. 1). At the moment of emission, the source and the observer are at the periphery of this sphere at the same distance from its center, and in the static world the exchange of photons between them does not lead to GRC. But in the expanding world, the photons emitted at a smaller radius of this sphere and stronger gravity are received by the observer at a larger radius at the same mass of the sphere, when the gravity on its surface has become weaker, which leads to the local GRC, depending on the difference in radii (Fig. 2). The influence of gravity of the matter of the outer region is similar and leads to the global GRC due to the difference in average densities, and hence the dilation of proper times, in two epochs. The photons from the observer to the source have the same two types of GRC in full agreement with the cosmological principle.

Thus, it is shown that for photons from a source resting relative to the observer, to the stretching of their wavelength during expansion (analogue of the linear Doppler effect), it becomes added GRC (analogue of the quadratic Doppler effect) and the total shift becomes similar to the relativistic Doppler effect. All this shows that GRC is not taken into account in the known relations of the Friedmann model, as it was commonly believed [4], and this effect must be taken into account separately both in the models and in the interpretation of observational data for distant objects.

In Section 1 a consistent method for accounting for GRC in cosmology and two of its types, the local and global GRC, are described. In Section 2 the consequences of GRC for cosmological models, interpretation of data, and also for CMB are studied. In Appendix, two former methods of accounting for gravitational shift are considered and their criticism is given.

\section{The theory of gravitational frequency shift in cosmology}

\subsection{A new method for accounting for gravity in cosmology and two types of GRC}

As noted above, the two former methods of taking into account the gravitational shift in a homogeneous medium (see Appendix) were based on the artificial selection of a certain area, a sphere centered at the source or at the observer, claiming that the propagation of photons from the source is affected only by the matter of this sphere, and as a result, both methods came to contradictions. Therefore, a third, consistent method without artificial assumptions of these methods is needed, which would ensure the equality of the source and the observer in the exchange of photons. 
As in the former methods, we first study the simplified case of non-expanding space with receding sources, where there is a Doppler shift. Then consider an expanding space with sources resting relative to the observer, when there is no Doppler shift, but there is an extension of wavelengths along the path.

For this, instead of the two opposite conditions of the former methods (spheres around the observer or the source), we select that area common for the observer and the source in their vicinity, through which the flux of photons passes and whose matter exactly affects the frequency of these photons. To do this, consider the following thought experiment. If we remove all matter in the sphere $\mathrm{C}$ between the observer and the source (Fig. 2), then in the static world the surrounding matter creates a constant gravitational potential inside this empty cavity. Since in such a static cavity the potential is the same everywhere, the photons move from the source to the observer (and vice versa) without gravitational shift.

Three conclusions follow from this fact. The first and most important conclusion is that in the static world, during the propagation of photons inside the cavity between the source and the observer, the gravity of matter outside the cavity does not change their initial frequency.

The second conclusion is that in the expanding world the gravitational potential created by the external matter inside the considered cavity changes with time due to decreasing of the matter density. As a result, during the propagation of photons in the cavity, the global GRC appears due to the influence of the outer matter. This shift is discussed below in Section 1.2.

And, finally, the third conclusion is that after taking into account the global GRC, the photon frequency is affected only by the gravity of matter inside the sphere between the observer and the source with the radius $r_{e} / 2$. This influence is easy to take into account, since both the source and the observer are on the surface of the sphere of the given mass. In the static world there will be no shift, but in the expanding world there will be a redshift due to the potential difference during the emission and registration of photons (Fig. 2). This type of gravitational shift is caused by matter in the vicinity and therefore it is a local GRC, which is discussed in Section 1.3.

Thus, the consistent method of accounting for the local GRC is based on considering the motion of photons in the field of a sphere between the source and the observer, when both of them are located at each moment on the periphery of this sphere at the same distance from its center, while the global GRC follows from taking into account the influence of the average density of matter in different epochs to the rate of proper times. At the exchanging of photons, the source and the observer are equivalent, since the trajectories of the photons in both directions are symmetric relative to the center of the sphere, which is in full agreement with the cosmological principle.

Below, the magnitude of both types of gravitational redshift in cosmology are calculated, and in Section 3 their consequences for theory and observational data are discussed.

\subsection{The global GRC.}

Let's the sphere between the observer and the source is an empty cavity (Fig. 2) and consider the gravitational potential created in it by the outer matter. In this cavity, we can introduce a static frame of reference with spherical coordinates centered at the center of the cavity and corresponding hypersurface of simultaneity $t=$ const. with world time $t$. The linear element is then contains some function of time $f(t)$, determined by the density $\rho(t)$ of outer matter:

$$
d s^{2}=f(t) d t-d r^{2}-r^{2} d \Omega^{2} .
$$

For a cavity inside a uniform dust ball with a finite surface radius, this function can be found from the Oppenheimer-Snyder solution. However, in cosmology this function will have to be determined from the observational data, which will be discussed in Section 2. Here, we express the frequency shift in terms of this function, assuming it to be known. 
In a static world with constant density $\rho=$ const., it would be $f=$ const., which allows to redefine world time as $f \cdot d t^{2}=d t^{\prime 2}$. Here $t^{\prime}$ remains be world time, since for simultaneous events its values will still be the same. In the static case, therefore, there is no global GRC.

In the expanding world, where the average density of matter during the propagation of photons from the source inside the cavity will decrease, and the radius of the cavity will increase (Fig. 2), the value $f(t)$ will increase by time and the gravitational dilation of proper times will weaken, i.e. $f\left(t_{2}\right)>f\left(t_{1}\right)$ at $t_{2}>t_{1}$. At the first stage, we will determine the value of GRC for this reason in its pure form, excluding the Doppler shift. To do this, consider the photons emitted by a source that, at emission, was at rest relative to the cavity's center, and let the observer at receiving these photons also be at rest relative to this center. Since $\tau_{o}=\Delta t \sqrt{f\left(t_{o}\right)}$ and $\tau_{e}=\Delta t \sqrt{f\left(t_{e}\right)}$, then for the global GRC we obtain:

$$
1+z_{g G}=\frac{\lambda_{o}}{\lambda_{e}}=\frac{\tau_{o}}{\tau_{e}}=\frac{\sqrt{f\left(t_{o}\right)}}{\sqrt{f\left(t_{e}\right)}} .
$$

At the second stage, we will take into account the Doppler shift and consider the emission and registration of photons by the source and observer comoving the surface of cavity. At the speed of expansion of the boundary of the cavity relative to its center $v_{r}=\mathrm{Hr}$, the relative speed $v$ of the source and the observer on the hypersurfaces of simultaneity $t_{e}=$ const. and $t_{o}=$ const. is determined by the relativistic formula, which in the simplest case, when $v_{r}$ weakly depends on time, gives:

$$
v=\frac{2 H r}{1+H^{2} r^{2}}, \quad 1+z_{D}=\sqrt{\frac{1+v}{1-v}}=\frac{1+H r}{1-H r}=\frac{1+H_{o} r_{o} / 2}{1-H_{o} r_{o} / 2},
$$

As the result, the total shift in this approximation takes the form:

$$
1+z_{g G, D}=\left(1+z_{g G}\right)\left(1+z_{D}\right)=\frac{\sqrt{f\left(t_{o}\right)}}{\sqrt{f\left(t_{e}\right)}} \cdot \frac{1+H_{o} r_{o} / 2}{1-H_{o} r_{o} / 2} .
$$

Thus, the global GRC arises due to the fact that the time component of the metric at the boundary of the cavity (or gravitational potential), created by the matter outside the cavity, is constant in space, but depends on time due to a decrease in the average density of matter during expansion. Photons are emitted at an epoch with a higher matter density and are received at an epoch with a lower density. Earlier, the gravity of denser matter slowed down its proper times stronger and photons were emitted with a lower frequency than now, which leads to GRC of "old" photons relatively "young" ones.

\subsection{The local GRC.}

To determine the local GRC in its pure form, consider the opposite to the considered in the previous Section case - let the cavity between the observer and the source contains dust matter, while there is no outer matter, i.e. let us consider the gravity of a ball in empty space.

In the static world, photons from a source at the periphery of the gravitating ball come to the observer at the diametrically opposite periphery at the same radius of its surface. Since there is no potential difference between these points, there will be no gravitational shift. Thus, unlike the previous two methods, which predicted redshift or violetshift even in the static world (see Appendix), in fact there is no gravitational shift at all.

In the expanding world, the gravitational shift at the periphery of the gravitating sphere will be studied firstly also without the Doppler shift, when the source at emission and the observer at reception do not comove the surface of the surface of the ball, but are at rest relative to the ball's center. Now, during the propagation of photons, the ball will expand and the photons emitted at a smaller radius will come to the observer at a larger radius, when its gravity 
is weaker. The ratio of the time components of the Schwarzschild metric (or the potential difference in the case of a weak field), will lead to the local GRC for the photon frequencies.

Thus, photons emitted at the radius of the ball $r\left(t_{e}\right)$ come to the observer at a larger radius $r\left(t_{o}\right)>r\left(t_{e}\right)$ at the same mass of the ball $M_{e} / 8$, which leads to the local GRC. In the case of non-expanding space and a source that was at rest relative to the observer, this gives:

$$
1+z_{g L}=\frac{\lambda_{o}}{\lambda_{e}}=\sqrt{\frac{1-2 G M(r) / r\left(t_{e}\right)}{1-2 G M(r) / r\left(t_{o}\right)}}=\sqrt{\frac{1-H_{e}^{2} r_{e}^{2} / 4}{1-H_{0}^{2} r_{o}^{2} / 4}} \simeq 1+\frac{1}{8}\left(H_{0}^{2} r_{o}^{2}-H_{e}^{2} r_{e}^{2}\right)
$$

Accordingly, the photons from the source, which in the observer's rest frame was moving away with a speed $v$, at the moment of emission contain the Doppler redshift $1+z_{D}$ as in (3) and therefore the combined redshift $1+z_{g L, D}$ will be equal to:

$$
1+z_{g L, D}=\left(1+z_{g L}\right)\left(1+z_{D}\right)=\frac{\lambda_{o}}{\lambda_{e}}=\frac{1+H_{o} r_{o} / 2}{1-H_{o} r_{o} / 2} \cdot \frac{\sqrt{1-H_{e}^{2} r_{e}^{2} / 4}}{\sqrt{1-H_{o}^{2} r_{o}^{2} / 4}} .
$$

Thus, the maximum value of the local GRC, which occurs at $r_{o} \gg r_{e}$, is approximately half of the quadratic Doppler redshift.

\subsection{Total GRC and its combinations with other types of redshift.}

Local and global GRC from (2) and (5) together form a total GRC $1+z_{g}$ as their product:

$$
1+z_{g}=\left(1+z_{g G}\right)\left(1+z_{g L}\right) \text {. }
$$

Taking into account the Doppler effect at the beginning of the path (3) gives the combined shift $1+z_{g, D}$ as:

$$
1+z_{g, D}=\left(1+z_{g}\right)\left(1+z_{D}\right) .
$$

At turning to the expanding space of general relativity with a source at rest relative to the observer, the Doppler shift of photons is replaced by stretching along the path of the photon wavelength with redshift due to stretching:

$$
1+z_{E}=\frac{a_{0}}{a} .
$$

The corresponding combined shift (stretching and gravity) $1+z_{g, E}$ is:

$$
1+z_{g, E}=\left(1+z_{g}\right)\left(1+z_{E}\right) .
$$

Here, GRC amplifies the redshift due to stretching by adding a quadratic part, similar to the quadratic Doppler effect. The photons from the observer come to the source with similar GRC, which leads to the equivalence of two emitting objects in the homogeneous world.

And finally, the combination of full GRC with both of the previous mechanisms of redshift, stretching and Doppler shift, gives:

$$
1+z_{g, E, D}=\left(1+z_{g}\right)\left(1+z_{E}\right)\left(1+z_{D}\right) .
$$

Here we will not dwell on the question of which of the combinations is used in various cosmological models and just note that in any of them, taking into account the full GRC is required. Some details of such accounting are discussed in the next Section. 


\section{Consequences of two types of GRC in cosmology}

\subsection{Consequences of GRC for cosmological models and interpretation of data}

In Section 1, it was shown that the theory of GRC can be formulated in a consistent form. In this Section, it will be shown in a brief form that taking into account of GRC introduces significant corrections both into the interpretation of observational data on distant objects and early epochs, and into cosmological models, by changing their parameters and predictions.

GRC sufficiently modifies the observational data on distant objects for which the quadratic in distance or velocity contributions are noticeable, or even basic. Depending on the interpretation of the linear part of the contributions to the redshifts as Doppler, stretching, or both, to interpret the data on redshifts in the nonlinear part, we obtain the combined redshifts $z_{g, D}$ from (8), $z_{g, E}$ from (10) or $z_{g, E, D}$ from (11).

In all these cases, the contribution of GRC to the quadratic part is comparable by the contribution of the known mechanisms, and therefore the estimates of the distances, and hence the absolute luminosities of the sources, will noticeably decrease.

Of the two types of gravitational shift, the theory of local GCS for photons from observable objects does not require new data in addition to those that follow from cosmological models Therefore, any of the models allows one to calculate the local GRC $z_{g, L}$ for objects at a given luminosity distance. This means that corrections for this part of GRC can be separated from the observed redshifts $z_{o b s}$. By labeling the contributions of other effects as $1+z_{E, D, \ldots}$, which in this case can also be calculated in principle, the remainder of the observed redshift $z_{o b s}$ can then be ascribed to the global GRC:

$$
1+z_{g G}=\frac{1+z_{o b s}}{\left(1+z_{g L}\right)\left(1+z_{E, D, \ldots}\right)} .
$$

The calculation of this global GRC $z_{g G}$ is a more difficult task, since it requires knowledge of the boundary conditions "at infinity", and hence knowledge of the global structure of the universe, which has always been problematic. Thus, the global GRC turns out to be the most sensitive to the choice of the cosmological model, and therefore, at distinguishing its contribution from others, for example, as pointed out above, this feature makes it a valuable tool for testing models of the universe.

In this regard, we note two possibilities. First, it will be possible to make a choice between the models of a closed universe with a finite volume for the observable universe and an open universe with an actually "infinite" volume. Secondly, for models with a finite volume, it will be possible to obtain additional information for the total mass of matter and the radius of curvature in different epochs, including the present one.

\subsection{Consequences of GRC for CMB and the early universe}

The ratio of the temperature of CMB at an earlier epoch $T_{e}$ to its observed temperature $T_{r}$ is determined by the ratio of the observed wavelength $\lambda_{r}$ to the wavelength at that epoch $\lambda_{e}$.

In models of Newtonian cosmology, taking into account kinematical relativistic corrections, the redshift is the result of the Doppler effect on hypersurfaces $t=$ const. and the temperature ratio for ultrarelativistic velocities $v=1-\delta v, \delta v \ll 1$ is given by the relation:

$$
1+z_{D}=\frac{\lambda_{r}}{\lambda_{e}}=\frac{T_{e}}{T_{r}}=\sqrt{\frac{1+v}{1-v}} \simeq \sqrt{\frac{2}{\delta v}} .
$$

Accordingly, taking into account the contribution of GRC, which is of the same order, leads to a combined redshift: 


$$
1+z_{g, D}=\left(1+z_{g}\right)\left(1+z_{D}\right)=\frac{\lambda_{r}}{\lambda_{e}}=\frac{T_{e}}{T_{r}} \sim \frac{2}{\delta v} .
$$

At $z_{g, D} \sim 1500(14)$ takes the form:

$$
z_{g, D} \simeq z_{g} z_{D} \simeq \frac{T_{e}}{T_{r}}=\frac{\lambda_{r}}{\lambda_{e}} \sim \frac{2}{\delta v}, \quad \delta v \simeq \frac{2}{z_{g, D}} \simeq 9 \cdot 10^{-7},
$$

and $z_{g} \sim z_{D} \sim \sqrt{1500} \sim 38$. In this case, the inhomogeneities in the recombination epoch, through which the relic flow passed, will be flattened for us due to the relativistic and gravitational contractions.

In the Friedmann model, where the redshift is attributed to stretching, the temperature ratio is given by the ratio of scale factors $a_{0} / a$, and therefore depends only on $z_{E}$, which gives the equation for $T(a)$ :

$$
\frac{T_{e}}{T_{r}}=\frac{\lambda_{r}}{\lambda_{e}}=\frac{a_{0}}{a}=1+z_{E}, \quad \frac{d T}{T}=-\frac{d a}{a} .
$$

Accounting for GRC leads to a combined redshift and temperature ratio:

$$
1+z_{g, E}=\frac{\lambda_{r}}{\lambda_{e}}=\frac{T_{e}}{T_{r}}=\left(1+z_{g}\right) \frac{a_{0}}{a}=\left(1+z_{g}\right)\left(1+z_{E}\right) .
$$

In both treatments, the relationship between redshifts and $a$ now turns out to be more complicated, since it is necessary to set $z_{g}(a)$. But the fact that $z_{g}$ depends on the ratio $a_{0} / a$ quadratically allows us to draw some conclusions. In contrast to linear stretching of wavelengths, GRC is an analogue of the quadratic Doppler effect dominating at large $z$. Therefore, GRC affects the properties of the CMB more strongly than linear stretching. At the same $a$ we have $z_{g} \gg z_{E}$, and therefore the interpretation of the observed redshift as a combination of the Doppler and GRC $z \sim z_{g, D}$ means that $a$ now refers to larger value than it followed from linear stretching, and therefore to a sufficiently later time $t$.

A larger value of $a$ and a later time $t$ when CMB was isolated from the matter does not mean that the densities of matter and radiation in the epoch when it was $a=a_{0} / z_{E}$, were different than previously assumed. Notice that it is also necessary to take into account the possibility of a faster rate of proper times in this epoch, which also leads to higher values of the energy densities of matter and radiation [7].

Thus, for radiation emitted in the early universe, the contributions of both types of GRCs are no longer different and significantly modify the previously accepted picture, changing the distance to sources, their age and evolution, as well as the properties of CMB.

\section{Conclusion}

Thus, the theory of GRC can be formulated in a consistent form, and it introduces rather serious corrections both into the cosmological models and their predictions, and into the interpretation of data.

Of the two types of GRC, the theory of local shift for photons from observable objects does not require new hypotheses and its consequences can therefore be used to correct the data in the form of corrections for this part of the effect.

The situation with the global GRC is more complicated, since its calculations require knowledge of the structure of the universe on a global scale. Since the global GRC is sensitive to the choice of the cosmological model, this feature makes it a valuable tool for testing cosmological models. In particular, it allows to consider in more detail the possibility of a closed universe and to determine its parameters. 
In the early universe, the contributions of both types of GRCs are no longer different and significantly modify the previous picture concerning the distances to objects, their age and evolution, as well as the properties of CMB.

All these consequences of both types of GRC, considered so far in general form, require further more detailed study and the results of such studies will be presented in subsequent publications.

\section{Appendix. Inconsistency of two former treatments of gravitational shift in cosmology}

In the two previous methods, the gravitational frequency shift in cosmology was first studied in the simplified case of sources receding in static space. Then the total shift is reduced to Doppler and gravitational shifts.

Depending on what part of the surrounding matter was considered to affect photons, there are three possible methods for calculating the gravitational shift. A consistent method that correctly solves the problem is described above in the article. Here we will consider the details of two former methods that were presented in the literature and were considered as conventional ones. They gave a gravitational shift with the opposite sign and therefore contradicted each other, while each of the methods contradicted the cosmological principle, and all this showed their inconsistency.

The confusion in the literature regarding these two methods was such that they were considered equivalent ones, regardless of the opposite of their predictions. In particular, the followers of the second method (see, for example, [5]) usually referred to the article [3], describing the first method, as a theoretical basis. Sometimes, while describing the physical picture by the first method, calculations and conclusions were made by the second method [6].

\section{The first method with a gravitating ball around the source}

In observational cosmology, at calculating the apparent luminosity, a sphere around the source is considered, onto which its radiation has spread, and it is clear that the gravity of matter in this sphere somehow affects the photons during their propagation. In the first treatment of gravitational shift in a homogeneous medium, proposed by Bondi in 1947 [3], the motion of photons was considered in the field of matter in just such a sphere around the source with a radius equal to the distance to the observer $r_{e}$ (sphere A in Fig. 1), and the effect of outer matter was neglected. The photons come from the center of the gravitating ball of mass $M_{e}$ to its periphery, and the gravitational redshift at their observation was predicted.

To calculate this type of shift in its pure form, consider photons from a source that was at rest relative to the observer at the moment of emission. The shift of the observed wavelength $\lambda_{o}$ relative to the wavelength $\lambda_{e}$ at the source is then given by the formula:

$$
1+z_{g}=\frac{\lambda_{o}}{\lambda_{e}}=\frac{1}{\sqrt{1-2 G M_{e} / r_{e}}}, \quad z_{g}>0 .
$$

The photons from the source receding from the observer with a speed $v$ contain the Doppler redshift $1+z_{D}$ at emission and the total shift $1+z$ is equal to:

$$
1+z=\left(1+z_{D}\right)\left(1+z_{g}\right)=\frac{\lambda_{o}}{\lambda_{e}}=\sqrt{\frac{1+v}{1-v}} \cdot \frac{1}{\sqrt{1-2 G M_{e} / r_{e}}} .
$$

In cosmology $v=H r$, where $H=\dot{a} / a$, as well as $M_{e}=4 \pi \rho_{e} r_{e}^{3} / 3$ and $8 \pi G \rho_{e} / 3=H_{e}^{2}$, which allows us to write (19) in the form:

$$
1+z=\frac{1+H_{e} r_{e}}{\sqrt{1-H_{e}^{2} r_{e}^{2}}} \cdot \frac{1}{\sqrt{1-H_{e}^{2} r_{e}^{2}}}=\frac{1}{1-H_{e} r_{e}} \approx 1+H_{e} r_{e}+H_{e}^{2} r_{e}^{2} .
$$


Thus, here the redshift due to the quadratic Doppler effect, equal in the first approximation to $H_{e}^{2} r_{e}^{2} / 2$, becomes doubled due to the gravitational redshift.

Consider now the photons that emitted by the former observer and received by the former source. Then these photons from the periphery of the sphere around the source fall into its center, and therefore, for observers at the source, the gravitational shift will be violetshift and will exactly compensate for the contribution of the quadratic Doppler effect:

$$
1+z=\frac{1+H_{e} r_{e}}{\sqrt{1-H_{e}^{2} r_{e}^{2}}} \cdot \sqrt{1-H_{e}^{2} r_{e}^{2}}=1+H_{e} r_{e} .
$$

Thus, the first method violates the cosmological principle, since in the exchange of photons between two equivalent objects the predicted gravitational shift has a different sign.

\section{The second method with a gravitating ball around the observer}

In the second treatment of gravitational shift in cosmology, similar in essence, but opposite in formulation of the problem and the result, which was presented by Zeldovich and Novikov (Ch. $3 \S 5$ [4]), the motion of photons was considered in the field of matter in a sphere around the observer with a radius up to the source $r_{o}$ (sphere B in Fig. 1) by neglecting the effect of outer matter. Then the photons come from the periphery of the sphere of mass $M_{o}$ to its center, and therefore the gravitational violetshift at the observer was predicted for them. This treatment was later widely used in the literature $[5,6]$ and has become regarded as a standard one.

The photons from the source which was at rest relative to the observer at the moment of emission, then have the shift:

$$
1+z_{g}=\frac{\lambda_{o}}{\lambda_{e}}=\sqrt{1-2 G M_{o} / r_{o}}, \quad z_{g}<0 .
$$

For photons from a receding source, the total shift will be:

$$
\begin{gathered}
1+z=\frac{\lambda_{o}}{\lambda_{e}}=\sqrt{\frac{1+v}{1-v}} \cdot \sqrt{1-2 G M_{o} / r_{o}}, \\
1+z=\frac{1+H_{e} r_{e}}{\sqrt{1-H_{e}^{2} r_{e}^{2}}} \cdot \sqrt{1-H^{2} r_{o}^{2}}=1+H r_{o}, \quad z=H r_{o} .
\end{gathered}
$$

i.e. the gravitational violetshift exactly compensates for the redshift from the quadratic Doppler effect, leaving only its linear part.

In this case, linear growth with distance $z=H r_{o}$ means that the total redshift is the same as when taking into account only the extension of the wavelength during expansion. This property was then considered as proof of the indistinguishability of the two treatments of the cosmological redshift - as a result of stretching of wavelengths during the expansion of space and as the Doppler effect when removing sources in non-expanding space, taking into account the gravitational field of the ball around the observer.

As in the first method, consider also the photons that propagate from the observer to the source. These photons from the center of the sphere around the observer reach the source at its periphery, and therefore for observers near this source, the shift will be redshift and doubles the contribution of the quadratic Doppler effect:

$$
1+z=\frac{1+H_{e} r_{e}}{\sqrt{1-H_{e}^{2} r_{e}^{2}}} \cdot \frac{1}{\sqrt{1-H_{e}^{2} r_{e}^{2}}}=\frac{1}{1-H_{e} r_{e}} .
$$

Thus, the second method also violates the cosmological principle, since in the exchange of photons between two equivalent objects, the gravitational shift has a different sign. Moreover, this sign is opposite to the sign that follows from the first method. 
We also note the misconception that the gravitational shift is allegedly taken into account in the well-known relations of the Friedmann model with stretching of wavelengths. This was proved by referring to the fact that the Newtonian approximation with the Doppler redshift and with the gravitational violetshift reproduces the first terms of the expansion of the relations of the Friedmann model ([4] Ch. 3, Sections 5, 12). However, then the relations of the Friedmann model are not reproduced for photons from the observer to the source, since the Doppler shift will be the same, while the gravitational shift, according to [4], will change sign and becomes redshift. The same situation is for the first method, which shows their internal inconsistency, as well as the fact that the proofs in them are not rigorous, but based on accidental coincidences in artificially selected special cases.

\section{References}

1. Einstein A. (1916) Ann. d. Phys., 1916, 49, 769

2. Okun L. B. et al (2000) Am. J. Phys. 68, 115; (1999) Phys. Usp. 42(10), 1045.

3. Bondi H. (1947) MNRAS, 107, 410.

4. Zeldovich Y. B., Novikov I. D. (1983) Rel. Astroph. v.2 Struct. \& Evol. Univ., U.Ch.P.

5. Peacock J. A. (1999) Cosmological physics, C.U.P; (2008) arXiv:0809.4573v1.

6. Whiting A. B. (2004) The Observ., 124, 174.

7. Zakir, Z. (2020) Quant. \& Grav. Phys., 1:008-7160; (2021) Quant. \& Grav. Phys., 2:012-7533 